\title{
A FIXED POINT CRITERION FOR COMPACT $T_{2}$-SPACES
}

\author{
LOUIS A. TALMAN
}

ABSTRACT. We prove a fixed point theorem which has as consequences some theorems of W. G. Dotson, Jr., [2], [3] and of K. Baron and J. Matkowski [1].

1. Introduction and definitions. In recent articles, W. G. Dotson, Jr., [2], [3] as well as K. Baron and J. Matkowski [1] have extended the Banach contraction principle to nonexpansive self-mappings of certain classes of compact metric spaces. The purpose of this paper is to present an extension of the methods of these authors to certain self-mappings of arbitrary compact $T_{2}$-spaces.

Let $X$ be a separated uniform space (see [5] for terminology and notation). A self-mapping of $X$ is any function from $X$ into itself. (Note that we do not require any continuity of a self-mapping.). The symbol $X^{X}$ will denote the space of all self-mappings of $X$ equipped with the topology of uniform convergence on $X$. When $\phi \in X^{X}$ and $F \subseteq X^{X}$, we will say that $\phi$ has fixed points modulo $F$ provided that, for each $f \in F$, at least one of the functions $\phi \circ f$ and $f \circ \phi$ has a fixed point.

We shall use the following Proposition, which is an easy consequence of the definitions.

Proposition. Let $\left\{x_{a}: \alpha \in \mathbb{Q}\right\}$ be a net in a uniform space, $X$, which converges to some point $x_{0} \in X$. If $\left\{f_{a}: a \in \mathbb{P}\right\}$ is a net in $X^{X}$ which converges to a uniformly continuous $f$ in $X^{X}$, then $\left\{f_{a}\left(x_{a}\right): \alpha \in \mathbb{Q}\right\}$ converges to $f\left(x_{0}\right)$ in $X$.

2. The main theorem. We are now ready to give the main theorem. First, recall that a compact $T_{2}$-space is, in a canonical way, a separated uniform space, so that the notions of the previous section are meaningful in such a space.

Theorem 1. Let $X$ be a compact $T_{2}$-space, and let $\phi$ be a continuous self-mapping of $X$. If there is a net $\left\{f_{\alpha}: \alpha \in \mathbb{Q}\right\}$ in $X^{X}$ such that (i) $\phi$ has

Received by the editors April 1, 1974.

AMS (MOS) subject classifications (1970). Primary 47H10,54H25; Secondary 47H99. 
fixed points modulo $\left\{f_{a}: a \in \mathbb{Q}\right\}$, and (ii) $\left\{f_{a}: a \in \mathbb{Q}\right\}$ converges to the identity function, $\mathrm{id}_{X}$, on $X$, then $\phi$ has a fixed point.

Proof. Condition (ii) requires that $\mathrm{id}_{X}$ be in the closure, in the topology on $X^{X}$, of the collection $F=\left\{f_{a}: \alpha \in \mathbb{Q}\right\}$. If we let $F_{R}=\left\{f_{a} \in F: f_{a} \circ \phi\right.$ has a fixed point $\}$ and $F_{L}=\left\{f_{a} \in F: \phi \circ f_{a}\right.$ has a fixed point $\}$, then, by (i), $F=$ $F_{R} \cup F_{L}$. Thus, we can select a subnet $\left\{f_{\beta}: \beta \in \mathbb{B}\right\}$ of $F$ which converges to $\mathrm{id}_{X}$ and is entirely contained within $F_{R}$ or $F_{L}$. In the first case, we define $\phi_{\beta}: X \rightarrow X$ for each $\beta \in \Re$ by $\phi_{\beta}(x)=f_{\beta}(\phi(x))$; in the second case, we define $\phi_{\beta}(x)=\phi\left(f_{\beta}(x)\right)$. In either case, for each $\beta \in \Re$, there is an $x_{\beta}$ in $X$ such that $\phi_{\beta}\left(x_{\beta}\right)=x_{\beta}$. Since $X$ is compact, we may assume that $\left\{x_{\beta}: \beta \in \mathscr{B}\right\}$ converges to some $x_{0}$ in $X$. But $\left\{\phi_{\beta}: \beta \in \mathfrak{B}\right\}$ converges to $\phi$ in $X^{X}$, so application of the Proposition yields that $x_{0}=\lim x_{\beta}=\lim \phi_{\beta}\left(x_{\beta}\right)=\phi\left(x_{0}\right)$, and $x_{0}$ is a fixed point of $\phi$.

3. Consequences. We now apply Theorem 1 in order to obtain some results due to Dotson and to Baron and Matkowski.

Theorem 2 (Dotson [3]). Let $S$ be a subset of a Banach space E, and equip $S$ with the relative topology from the weak topology on E. Suppose that $S$, so equipped, is compact, and that there exists a continuous function $F$ : $S \times[0,1] \rightarrow S$ such that (i) $F(s, 1)=s$ for every $s$ in $S$, and (ii) there is $a$ self-mapping, $\phi$, of ]0, 1 [ such that for every $s_{1}, s_{2}$ in $S$ and for all $t$ in ]0, $1[$ we have

$$
\left\|F\left(s_{1}, t\right)-F\left(s_{2}, t\right)\right\| \leq \phi(t)\left\|s_{1}-s_{2}\right\|
$$

Then any continuous function $\psi: S \rightarrow S$ which is nonexpansive with respect to $\|\cdot\|$ has a fixed point.

Proof. For each $n=1,2, \ldots$, let $t_{n}=n /(n+1)$ and define $\phi_{n}: S \rightarrow S$ by $\phi_{n}(s)=F\left(s, t_{n}\right)$. It is easy to see that $\left\{\phi_{n}\right\}$ converges in $s^{s}$ to id ${ }$. All that remains is to show that any $\psi$ which satisfies the hypotheses of the theorem must have fixed points modulo $\left\{\phi_{n}\right\}$. To this end, let $s_{1}, s_{2}$ be in $S$. Then for any $n=1,2, \ldots$, we have

$$
\left\|\phi_{n}\left[\psi\left(s_{1}\right)\right]-\phi_{n}\left[\psi\left(s_{2}\right)\right]\right\| \leq \phi\left(t_{n}\right)\left\|\psi\left(s_{1}\right)-\psi\left(s_{2}\right)\right\| \leq \phi\left(t_{n}\right)\left\|s_{1}-s_{2}\right\| .
$$

Since $\phi\left(t_{n}\right)<1$ for every $n$, we see that $\phi_{n} \circ \psi$ is a contraction on $S$ relative to the norm. But $S$ is weakly compact, hence norm closed, and, therefore, norm complete. By the Banach contraction principle, $\psi_{n} \circ \phi$ has a fixed point.

Theorem 2 yields as a corollary a fixed point theorem for nonexpansive 
self-mappings of compact metric spaces which was first proved by Dotson[3] for nonconvex subsets of Banach spaces.

Corollary. Let $X$ be a compact metric space with metric $\rho$, and suppose that there is a continuous function $F: X \times[0,1] \rightarrow X$ such that (i) $F(x, 1)$ $=x$ for every $x$ in $X$, and (ii) there is a self-mapping, $\phi$, of $] 0,1[$ such that for every $x_{1}, x_{2}$ in $X$ and for all $t$ in ]0, 1[ we have

$$
\rho\left(F\left(x_{1}, t\right), F\left(x_{2}, t\right)\right) \leq \phi(t) \rho\left(x_{1}, x_{2}\right) .
$$

Then any nonexpansive self-mapping of $X$ has a fixed point.

Proof. It is well known that any metric space can be embedded isometrically in a Banach space (see, e.g., [4, XII, 5.2, p. 286]). Thus, we may consider $X$ to be a compact subset of a Banach space $E$ with metric induced by a norm $\|\cdot\|$. The compactness of $X$ guarantees that the norm topology coincides with the weak topology on $X([4, \mathrm{XI}, 2.1, \mathrm{p} .226]$ or $[5,5.8, \mathrm{p} .141])$, and the conclusion follows from Theorem 2.

Following Baron and Matkowski $[1]$, we say that a metric space $(X, \rho)$ is an $S$-space if there exists an $x_{0}$ in $X$ such that for every $t$ in $] 0,1[$ there is a $\rho$-contractive self-mapping $f_{t}$ of $X$ for which the inequality $\rho\left(f_{t}(x), x\right)$ $\leq(1-t) \rho\left(x_{0}, x\right)$ holds for every $x$ in $X$.

Theorem 3 (Baron and Matkowski [1]). Every nonexpansive self-mapping of a compact S-space has a fixed point.

Proof. Let $(X, \rho)$ be a compact $S$-space, and let $F$ be a nonexpansive self-mapping of $X$. If the interval ]0, 1 [ is equipped with its usual order, then $\left\{f_{t}: t \in\right] 0,1[\}$, where $f_{t}$ is as in the definition of $S$-space, is a net in $X^{X}$ which converges to $\mathrm{id}_{X}$. Moreover, $f_{t} \circ F$ is easily seen to be a contraction on $X$ for each $t$ in $] 0,1\left[\right.$, so $F$ has fixed points modulo $\left\{f_{t}: t \in\right] 0,1[\}$.

\section{BIBLIOGR APHY}

1. K. Baron and J. Matkowski, A fixed point theorem for non-expansive mappings on compact metric spaces, Publ. Inst. Math. 15 (29) (1973), 25.

2. W. G. Dotson, Jr., Fixed point theorems for non-expansive mappings on starshaped subsets of Banach spaces, J. London Math. Soc. (2) 4 (1971/72), 408-410. MR 45 \#5837.

3. - On fixed points of nonexpansive mappings in nonconvex sets, Proc. Amer. Math. Soc. 38 (1973), 155-156. MR 47 \#2446.

4. J. Dugundji, Topology, Allyn and Bacon, Boston, Mass., 1966. MR 33\#1824. $16,1136$.

5. J. L. Kelley, General topology, Van Nostrand, Princeton, N. J., 1955. MR 